\title{
Genetic Transformation of Oat Mediated by Agrobacterium is enhanced with Sonication and Vacuum Infiltration
}

\author{
Nagesh Dattgonde, Sharad Tiwari ${ }^{*}$, Swapnil Sapre, Iti Gontia-Mishra \\ Biotechnology Centre, Jawaharlal Nehru Agriculture University, Jabalpur, India \\ * Corresponding author: Sharad Tiwari, Biotechnology Centre, Jawaharlal Nehru Agriculture University, Jabalpur, India. Phone: +919424658241; Fax: \\ +917612681021. E-mail: dirbcjbp.jnkvv-mp@nic.in
}

\begin{abstract}
Background: Oat (Avena sativa) with high nutritive value and fiber content is used as the main food grain in many countries for human diet as well as animal feed. Recently, it became difficult to transfer new genes through the conventional breeding due to the lack of desirable traits.

Objectives: The current study aimed at achieving a standardized protocol for Agrobacterium-mediated transformation in oat.

Materials and Methods: For oat transformation, mature seeds were sterilized, germinated, and used for explants generation. Agrobacterium tumefaciens GV3101 with the binary vector pCAMBIA 1305.1, which carries gus as reporter gene, was utilized in the transformation. The co-cultivation treatment assisted with sonication, and vacuum infiltration, and their combination was employed for transformation with different incubation periods of 48, 72, and 96 hours under the dark conditions.

Results: Among the different transformation treatments, the vacuum treatment with 72 hours dark incubation had the best results. Vacuum infiltration of the cultures from leaf base produced a maximum of $25 \%$ hygromycin-resistant explants. These explants upon GUS assay and PCR analysis revealed $21.85 \%$ and $19.04 \%$ transformation efficiency, respectively.

Conclusions: It could be concluded that vacuum infiltration assisted Agrobacterium-mediated transformation is the most efficient method to conduct the genetic improvement of the oat using transformation protocol.
\end{abstract}

Keywords: Avena; Agrobacterium; Sonication; Vacuum

\section{Background}

Oat is grown as a multi-purpose crop; it is used as a grain, forage, and a rotation crop. It has rich nutritional value, since it contains the soluble fibers with hypocholesterolemic properties (1). It has excellent growth habit, rapid recovery after cutting, and excellent quality fodder. In India, the yield of oat is quite low, compared with other cereal crops, owing to various environmental stresses. A number of efforts are made towards the development of new cultivars of oat with an improved disease, pest, and herbicide tolerance. The genetic improvement of the commercially important oat cultivars through the conventional breeding is arduous, slow, and costly. A huge improvement is noticed in several monocots by incorporating various agronomical traits using genetic engineering $(2,3)$. The development of a robust regeneration protocol is necessary to transform cereals (4). Agrobacterium-mediated transformation is a complex procedure controlled by diverse parameters of the bacterial, host, and environmental origin. By this technique of genetic transformation, a wide range of characters such as improvement of the plant tolerance to the environmental stresses, disease resistance, enhanced crop productivity, pest resistance, phytoremediation, and improved nutritional content of the crop plants are developed (5). To increase the transformation efficacy in the plants, sonication and vacuum infiltration play an important role $(6,7)$. Sonication produces microwounds by cavitation throughout the surface of tissue whereas vacuum infiltration provides a rapid entry of the Agrobacterium into the plant tissue. There are several reports of the enhanced genetic transformation using these techniques in various crops namely radish (8), citrus (9), cowpea (10), banana (6), lentil (11), and sugarcane (7). Gasparis et al., (12) reported Agrobacterium-mediated transformation of the oat using immature embryo and leaf explants. In the current investigation, efforts were made to improve the efficiency of the Agrobacterium-mediated 
transformation in oat by a conjoint effect of sonication and vacuum infiltration.

\section{Objectives}

The current study aimed at generating a proficient gene transfer system in oat cv. JO-1 using sonication-assisted Agrobacterium-mediated transformation (SAAT) and the vacuum-infiltration-assisted Agrobacteriummediated transformation (VIAAT). The influence of different explants, sonication, and vacuum infiltration were evaluated in Agrobacterium-mediated genetic transformation of oat cv. JO-1.

\section{Materials and Methods}

\subsection{Sterilization and Germination of Oat Seeds}

Seeds of cultivar JO-1 were procured from the All India Coordinated Forage Research Project (ICAR), Department of Agronomy, JNKVV Jabalpur. Initially, oat seeds were rinsed thoroughly with water. Further, the seeds were kept in $1 \%$ Tween-20 solution for 20 minutes and then treated for two minutes with $70 \%$ ethanol. The seeds were again treated with $0.5 \% \mathrm{HgCl}_{2}$ solution for five minutes. Finally, seeds were germinated on the Murashige and Skoog (MS) basal medium (13). The six-day-old seedlings were used to obtain leaf base explants, whereas mature embryos were excised from overnight soaked seeds under aseptic conditions.

3.2. Screening of the Explants for Antibiotic Sensitivity The explants were inoculated into the MS medium as a control, as well as different concentrations of the hygromycin ( 5 to $50 \mathrm{mg} . \mathrm{L}^{-1}$ ) and cefotaxime (50 to 750 $\mathrm{mg} . \mathrm{L}^{-1)}$ to assess antibiotic sensitivity.

\subsection{Agrobacterium Culture}

For genetic transformation studies, Agrobacterium tumefaciens strain GV3101 with plasmid $p C A M B I A 1305.1$ (11846 base pair) harboring gus ( $\beta$ glucuronidase) gene and catalytic intron under the regulation of CaMV35S promoter and nos terminator was used. The vector also had hptII (hygromycin phosphotransferase II) gene for plant selection with CaMV35S promoter and polyA terminator (Fig. 1A). A. tumefaciens was inoculated into a 50-mL Luria Bertani (LB) medium supplemented with rifampicin and kanamycin (50 mg. $\mathrm{L}^{-1}$ each) and incubated at $28^{\circ} \mathrm{C}$ for 16 hours at $200 \mathrm{rpm}$. Agrobacterium culture of OD 0.6 (at wavelength of $600 \mathrm{~nm}$ ) were centrifuged for five minutes at $28000 \mathrm{~g}$ and the bacterial cells' pellet was dissolved in the same amount of the co-cultivation broth (1X B $\mathrm{B}_{5}$ medium, 3\% sucrose, $100 \mathrm{mg} . \mathrm{L}^{-1} \mathrm{MES}, 100 \mu \mathrm{M}$ acetosyringone, $1 \mathrm{mg} . \mathrm{L}^{-1} \mathrm{GA}_{3}, 1.67 \mathrm{mg} . \mathrm{L}^{-1} \mathrm{BAP}, 400$ mg.L. ${ }^{-1}$ L-cysteine, $248 \mathrm{mg} . \mathrm{L}^{-1} \mathrm{Na}$-thiosulphate, and 154 mg.L ${ }^{-1}$ DTT).

\subsection{Sonication-assisted Agrobacterium-mediated} Transformation

For SAAT (17), calli developed from embryo and leaf base (Fig. 1B) were sonicated in a bath sonicator (HFfrequency: $35 \mathrm{KHz}$ ) submerged in $20 \mathrm{~mL}$ co-cultivation medium suspended with Agrobacterium for 30 seconds. These calli were placed at $25 \pm 2^{\circ} \mathrm{C}$ for 3-4 days in the dark conditions on a medium combination similar to cocultivation medium with 8 g.L $\mathrm{L}^{-1}$ agar, but without Agrobacterium.

\subsection{Vacuum-infiltration-assisted Agrobacterium- mediated transformation}

During co-cultivation, explants submerged in cocultivation medium with Agrobacterium were exposed to vacuum infiltration for 15 minutes.

3.6. Sonication and Vacuum-Infiltration-assistedAgrobacterium-mediated Transformation

Conditions optimized in SAAT and VIAAT were applied in combination with achieve maximum Agrobacterium-mediated transformation efficiency (8, 9).

\subsection{Selection and Plant Regeneration}

The calli were washed with MS broth supplemented with cefotaxime ( $\left.250 \mathrm{mg} \cdot \mathrm{L}^{-1}\right)$. The calli were blot dried on sterile filter paper before placing them on MS medium containing hygromycin $20 \mathrm{mg} . \mathrm{L}^{-1}$ and cefotaxime $500 \mathrm{mg} . \mathrm{L}^{-1}$. Calli showing growth and morphogenesis (Fig. 1C and D) on $20 \mathrm{mg} . \mathrm{L}^{-1}$ hygromycin supplemented MS medium were considered as putatively transformed plants. These calli were three times sub-cultured on hygromycincontaining medium at 10-12 days intervals.

\subsection{GUS Histochemical Assay}

Histochemical GUS analysis in explants was assayed after the third round of selection on antibiotics as described by Jefferson (14). Briefly, 10 explants with shoots were incubated in X-Gluc solution for overnight at room temperature under dark conditions, and washed with ethanol to remove chlorophyll. Histochemical localization of GUS activity was examined under stereomicroscope (Fig. 1E and F).

3.9. PCR Analysis of the Putative Transgenic Plants The genomic DNA was isolated from the putative transformed plant tissue as per Saghai-Maroof et al., (15). PCR amplification of the marker genes: $h p t I$ and the CAMV $35 S$ promoter region were performed to confirm the transformation as described by Sapre et al. (16) The amplification products of the hptII gene (499 bp) and CaMV $35 \mathrm{~S}$ promoter ( $500 \mathrm{bp}$ ) were subjected to the electrophoresis and compared with those of the positive control (plant transformation vector; i e, pCAMBIA plasmid) and negative control (DNA from non-transformed plants) (Fig. 2A and B). 

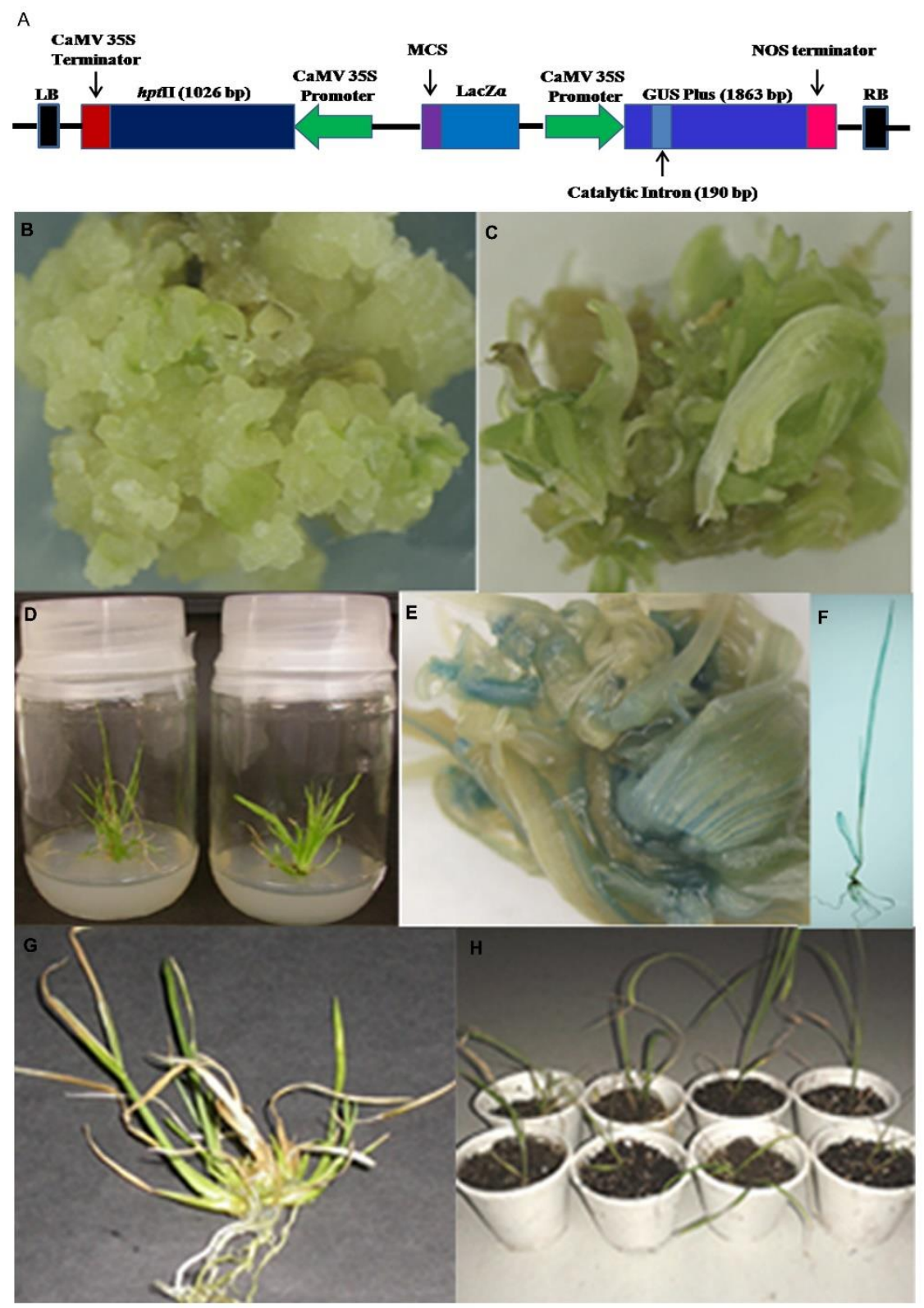

Figure 1. Regeneration and transgenic plant selection of oat; (A) pCAMBIA 1305 vector map; (B) Embryogenic callus from mature embryo explants; (C) Organogenesis from callus; (D) Green plantlets; (E, F) Expression of the gus gene in different tissues; (G, H) Plant with roots

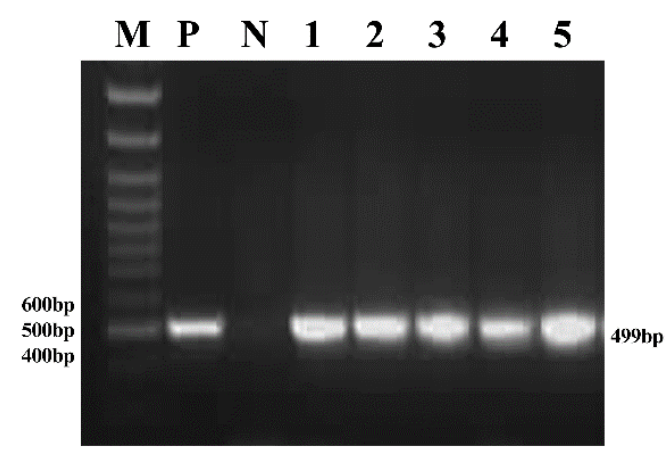

A

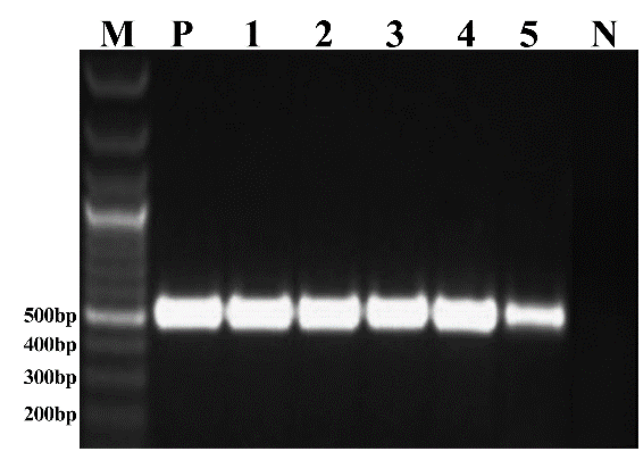

B

Figure 2. PCR Analysis of the putative transformed oat plants; A) PCR amplification of hptII gene; B) PCR amplification of the gus gene; lane M, 100 bp DNA ladder; P, positive control; lanes 1-5, putative transformants; N, non-transformed control plant 


\section{Results}

\subsection{Effect of Hygromycin and Cefotaxime}

The sensitivity of the explants to the antibiotic hygromycin was established prior to the actual transformation experiments by finding the minimal inhibitory concentration (MIC) to select presumed transformants. In the absence of antibiotics, the explants regenerated normally and produced calli and shoots; however, morphogenic ability of the cultures was inhibited with the addition of $20 \mathrm{mg} . \mathrm{L}^{-1}$ hygromycin, since adventitious shoots exhibited very slow growth. While, $25 \mathrm{mg} . \mathrm{L}^{-1}$ hygromycin inhibited shoot regeneration and resulted in the whitening of the cultures within 1-2 weeks. Explants dried due to necrosis within one week of the culture, when cultured on higher concentrations of hygromycin (30-40 mg.L $\left.\mathrm{L}^{-1}\right)$. Hence, MS medium fortified with $20 \mathrm{mg} . \mathrm{L}^{-1}$ of hygromycin was used to select the transformants in the subsequent transformation experiments.

\subsection{Influence of co-Cultivation Duration}

Duration of co-cultivation was influential on transformation efficiency. The 72-hour duration explants produced $13.87 \%$ (embryo) and $14.53 \%$ (leaf base) hygromycin-resistant cultures. The transformation efficiency at 72 hours was significantly higher compared with those of the 48 and 96 hours. These antibiotic-resistant cultures revealed the transformation efficiency of $7.36 \%$ and $6.71 \%$ in GUS histochemical assay and $7.30 \%$ and $6.09 \%$ in the PCR analysis of the transformation efficiency from the embryo and leaf-base explants, respectively (Table 1).

Table 1. Overall effects of co-cultivation period and different treatments on transformation efficiency

\begin{tabular}{|c|c|c|c|c|}
\hline \multirow[t]{2}{*}{ Total Number of Cultures } & \multirow[t]{2}{*}{ Explant } & \multicolumn{3}{|c|}{$\begin{array}{ll}\text { Transformation Efficiency, \% } \\
\end{array}$} \\
\hline & & Hygromycin-resistant Culture & GUS Putative Culture & PCR Putative Culture \\
\hline \multicolumn{5}{|l|}{ Sonication time (hour) } \\
\hline \multicolumn{5}{|l|}{48} \\
\hline 100 & Embryo & $22.59 \pm 1.23^{\mathrm{d}}$ & $15.56 \pm 0.87^{\mathrm{e}}$ & $14.78 \pm 1.28^{\mathrm{e}}$ \\
\hline 102 & Leaf base & $21.00 \pm 0.98^{\mathrm{e}}$ & $14.81 \pm 1.28^{f}$ & $13.11 \pm 1.61^{\mathrm{gh}}$ \\
\hline \multicolumn{5}{|l|}{72} \\
\hline 75 & Embryo & $24.19 \pm 0.87^{\mathrm{b}}$ & $19.78 \pm 1.16^{\mathrm{b}}$ & $19.26 \pm 1.59^{\mathrm{b}}$ \\
\hline 79 & Leaf base & $23.22 \pm 1.03^{c}$ & $18.70 \pm 1.05^{\mathrm{c}}$ & $17.58 \pm 1.29^{c}$ \\
\hline \multicolumn{5}{|r|}{ 年 } \\
\hline 68 & Embryo & $18.11 \pm 1.76^{j}$ & $14.59 \pm 0.93^{\mathrm{fg}}$ & $13.67 \pm 1.11^{\mathrm{f}}$ \\
\hline 73 & Leaf base & $19.89 \pm 0.76^{f}$ & $14.33 \pm 0.73^{\mathrm{fg}}$ & $12.89 \pm 1.26^{\mathrm{gh}}$ \\
\hline \multicolumn{5}{|l|}{ Vacuum infiltration } \\
\hline \multicolumn{5}{|l|}{48} \\
\hline 102 & Embryo & $15.93 \pm 0.98^{\mathrm{hi}}$ & $11.00 \pm 0.81^{\mathrm{k}}$ & $10.15 \pm 1.18^{j}$ \\
\hline 110 & Leaf base & $14.44 \pm 0.91^{\mathrm{k}}$ & $11.89 \pm 0.99^{j}$ & $10.67 \pm 1.02^{j}$ \\
\hline \multicolumn{5}{|l|}{72} \\
\hline 104 & Embryo & $24.33 \pm 0.83^{b}$ & $21.96 \pm 1.18^{\mathrm{a}}$ & $20.74 \pm 1.08^{a}$ \\
\hline 85 & Leaf base & $25.00 \pm 0.96^{\mathrm{a}}$ & $21.85 \pm 1.65^{\mathrm{a}}$ & $19.04 \pm 0.96^{\mathrm{b}}$ \\
\hline \multicolumn{5}{|r|}{ - } \\
\hline 96 & Embryo & $17.37 \pm 1.21^{\mathrm{i}}$ & $13.67 \pm 1.41^{\mathrm{hi}}$ & $12.78 \pm 0.84^{\mathrm{h}}$ \\
\hline 93 & Leaf base & $18.89 \pm 1.17^{\mathrm{g}}$ & $13.56 \pm 1.29^{\mathrm{hi}}$ & $11.33 \pm 0.92^{\mathrm{i}}$ \\
\hline \multicolumn{5}{|c|}{ Sonication + vacuum infiltration } \\
\hline \multicolumn{5}{|c|}{48} \\
\hline 89 & Embryo & $20.22 \pm 0.88^{f}$ & $14.30 \pm 1.16^{\mathrm{fg}}$ & $13.85 \pm 0.94^{\mathrm{f}}$ \\
\hline 84 & Leaf base & $20.00 \pm 0.71^{f}$ & $14.11 \pm 0.88^{\mathrm{gh}}$ & $13.33 \pm 0.78^{\mathrm{fg}}$ \\
\hline \multicolumn{5}{|l|}{72} \\
\hline 86 & Embryo & $21.41 \pm 1.10^{\mathrm{e}}$ & $17.67 \pm 0.75^{\mathrm{d}}$ & $16.30 \pm 0.71^{\mathrm{d}}$ \\
\hline 102 & Leaf base & $21.44 \pm 0.89^{\mathrm{e}}$ & $18.70 \pm 0.87^{\mathrm{c}}$ & $17.44 \pm 1.21^{\mathrm{c}}$ \\
\hline \multicolumn{5}{|c|}{ (1) } \\
\hline 114 & Embryo & $17.78 \pm 1.21^{\mathrm{hi}}$ & $13.22 \pm 0.82^{\mathrm{i}}$ & $13.07 \pm 0.65^{\mathrm{gh}}$ \\
\hline 112 & Leaf base & $16.30 \pm 1.56$ & $12.11 \pm 1.20^{j}$ & $11.22 \pm 0.98^{\mathrm{i}}$ \\
\hline \multicolumn{5}{|l|}{ Control } \\
\hline \multicolumn{5}{|l|}{48} \\
\hline 103 & Embryo & $11.30 \pm 1.37^{\mathrm{m}}$ & $9.09 \pm 0.78^{1}$ & $8.38 \pm 1.05^{k}$ \\
\hline 95 & Leaf base & $10.69 \pm 0.98^{n}$ & $8.78 \pm 0.72^{1}$ & $7.03 \pm 0.89^{1}$ \\
\hline \multicolumn{5}{|l|}{72} \\
\hline 99 & Embryo & $13.87 \pm 1.05^{1}$ & $7.36 \pm 0.65^{\mathrm{m}}$ & $7.30 \pm 0.71^{1}$ \\
\hline 98 & Leaf base & $14.53 \pm 1.13^{\mathrm{k}}$ & $6.71 \pm 0.54^{\mathrm{n}}$ & $6.09 \pm 0.69^{\mathrm{m}}$ \\
\hline \multicolumn{5}{|c|}{ 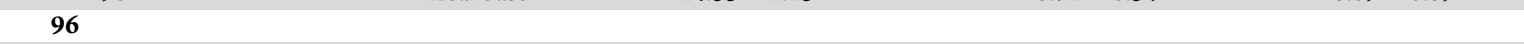 } \\
\hline 96 & Embryo & $9.02 \pm 0.86^{\mathrm{op}}$ & $4.90 \pm 0.43 \mathrm{o}^{\mathrm{p}}$ & $3.94 \pm 0.94^{\mathrm{n}}$ \\
\hline 94 & Leaf base & $7.48 \pm 0.73^{q}$ & $4.04 \pm 0.68^{\mathrm{P}}$ & $3.56 \pm 0.75^{\mathrm{n}}$ \\
\hline
\end{tabular}

Data are expressed as mean values of three independent experiments \pm standard deviation; means followed by the same letters are not significant at $5 \%$ level. 


\subsection{Efficacy of SAAT}

Under SAAT technique, cultures were subjected to ultrasound and inoculated in the Agrobacterium culture. With this technique, embryos and leaf base explants generated $24.19 \%$ and $23.22 \%$ hygromycin-resistant cultures after 72 hours of the co-cultivation, respectively. Analysis of the transformation efficiency on the embryo cultures showed the efficiency rates of $19.78 \%$ and $19.26 \%$ using GUS assay and gene specific PCR, respectively. Similarly, leaf base cultures responded to $18.70 \%$ and $17.58 \%$ transformation efficiency, according to GUS assay and PCR analysis, respectively (Table $\mathbf{1}$ ).

\subsection{Effect of VIAAT on Transformation Efficiency}

Vacuum pressure for 15 minutes at $750 \mathrm{mmHg}$ produced $24.33 \%$ and $25 \%$ hygromycin-resistant cultures in the embryo and leaf bases, respectively. The embryo explants exhibited $21.96 \%$ and $20.74 \%$ of the transformation efficiencies when analyzed on the basis of GUS assay and gene specific PCR analysis, respectively. Whereas, the leaf base explants upon GUS and PCR analysis produced $21.85 \%$ and $19.04 \%$ transformation efficiency, respectively (Table 1 ). 4.5 Effect of SVAAT on Transformation Efficiency

Upon the employment of both sonication and vacuum techniques, $21.4 \%$ of the cultures from embryos and leaf base survived on hygromycin after 72 hours of cocultivation. On the basis of histochemical GUS assay and gene specific PCR, the embryo explants exhibited $17.67 \%$ and $16.30 \%$ transformation efficiency, respectively. Whereas, $18.70 \%$ and $17.44 \%$ transformation efficiency was conferred by the leaf explants on the basis of GUS histochemical assay and PCR analysis, respectively (Table $\mathbf{1}$ ).

\section{Discussion}

The genetic and physiological status of the cells varies in different explants. However, these features affect the transformation efficiency. Along with explant, the cocultivation period of the cultures with Agrobacterium to assist transfer of T-DNA into plant cell also influences the efficiency of transformation. Determination of competence for transformation at different cocultivation duration, two explants namely mature embryos and leaf base were utilized. In the current study, the maximum transformation efficiency was obtained for the mature embryo explants at 72 hours of cocultivation duration. The study results were comparable with those of the transformation studies conducted on oat cultivars-cv. Bajka showing 12.3\% transformation efficiency using immature embryo as the explant (12). Co-cultivation duration of three days was sufficient for an efficient transformation. The longer co-cultivation duration resulted in a higher transient expression of the transgene in the soybean (17).

Agrobacterium-mediated transformation assisted with sonication was effective in the transfer of foreign DNA into cells in a number of species, especially those where the host was recalcitrant to the Agrobacteriummediated transformation (17).

Employment of embryos and leaf base explants produced $24.19 \%$ and $23.22 \%$ hygromycin-resistant cultures respectively, by the employment of SAAT followed by 72 hours of the co-cultivation period. The improved transformation via SAAT could possibly be due to micro-wounding on the surface of tissue, which aided in the entry of Agrobacterium into the plant tissue. The enhanced level of transient expression of the gus gene in soybean was observed by treating embryogenic cultures with sonication for 30 seconds (18).

The duration of the co-cultivation period after sonication had enhanced effects on the transformation efficiency.

Creation of vacuum may cause the release of gases from the culture probably through wounds instead of stomata. This phenomenon exposes more plant cells to the Agrobacterium, making it more susceptible to the transformation compared with the one where Agrobacterium is present in the culture superficially. The current study results suggested that the utilization of vacuum infiltration remarkably increased the transformation efficacy than the other treatments. VIAAT technique is reported for the efficient transformation of rice (19) and wheat (20), as well.

When both sonication and vacuum were applied simultaneously, more than $21 \%$ of the hygromycinresistant cultures were obtained in both explant types. The lower transformation efficiencies of SVIAAT method in comparison with those of the individual effect of processes (i e, SAAT and VAAT) may be attributed to the damage of the callus's tissue due to sonication followed by vacuum infiltration, hence the plant cells could not recover the incurred damage. The current study results were in congruence with those of the Subramanyam et al. (6), where the transformation of banana was conducted using SVAAT.

\section{Conclusions}

The current study results demonstrated a promising protocol for Agrobacterium-mediated genetic transformation in the oat. To date, this is the only maiden report of the oat transformation using SAAT and VIAAT. Duration of co-cultivation, sonication, and vacuum infiltration positively influence the oat transformation. The developed transformation protocol involves co-cultivation of $A$. tumefaciens along with cultures developed from leaf base under vacuum (15 inch $\mathrm{Hg}$ ) for 10-15 minutes in the presence of $100 \mu \mathrm{M}$ acetosyringone. The selection of transformant was conducted on MS medium supplemented with $20 \mathrm{mg} / \mathrm{L}$ hygromycin. This transformation protocol could be used efficiently to improve oat considering different agronomical and nutritive traits via genetic engineering. 


\section{Acknowledgement}

Sharad Tiwari appreciates the research grant from Madhya Pradesh Council of Science \& Technology, Bhopal, India.

\section{References}

1. Ferdman B, Singh G. Persistent Truncus Arteriosus. Curr Treat Options Cardiovasc Med. 2003;5(5):429438. pmid: 12941211

2. Jakob K, Zhou F, Paterson AH. Genetic improvement of $\mathrm{C} 4$ grasses as cellulosic biofuel feedstocks. Biofuels. New York: Springer; 2009. p. 113-138.

3. Takahashi W, Takamizo T. Molecular Breeding of Grasses by Transgenic Approaches for Biofuel Production. In: Ciftci Y, editor. Transgenic PlantsAdvances and Limitations. Rijeka: InTech; 2012. p. 91116.

4. Gless C, Lorz H, Jahne-Gartner A. Establishment of a highly efficient regeneration system from leaf base segments of oat (Avena sativa L.). Plant Cell Rep. 1998;17(6-7):441-445. doi: 10.1007/s002990050422 pmid: 30736616

5. Ziemienowicz A. Agrobacterium-mediated plant transformation: Factors, applications and recent advances. Biocatal Agricult Biotechnol. 2014;3(4):95102. doi: 10.1016/j.bcab.2013.10.004

6. Subramanyam K, Subramanyam K, Sailaja KV, Srinivasulu M, Lakshmidevi K. Highly efficient Agrobacterium-mediated transformation of banana cv. Rasthali (AAB) via sonication and vacuum infiltration. Plant Cell Rep. 2011;30(3):425-436. doi: 10.1007/s00299-010-0996-4 pmid: 21212957

7. Mayavan S, Subramanyam K, Arun M, Rajesh M, Kapil Dev G, Sivanandhan G, et al. Agrobacterium tumefaciens-mediated in planta seed transformation strategy in sugarcane. Plant Cell Rep. 2013;32(10):1557-1574. doi: 10.1007/s00299-0131467-5 pmid: 23749098

8. Park BJ, Liu Z, Kanno A, Kameya T. Transformation of radish (Raphanus sativus $L$.) via sonication and vacuum infiltration of germinated seeds with Agrobacterium harboring a group 3 LEA gene from B. napus. Plant Cell Rep. 2005;24(8):494-500. doi: 10.1007/s00299-0050973-5 pmid: 15843933

9. de Oliveira ML, Febres VJ, Costa MG, Moore GA, Otoni WC. High-efficiency Agrobacterium-mediated transformation of citrus via sonication and vacuum infiltration. Plant Cell Rep. 2009;28(3):387-395. doi: 10.1007/s00299-008-0646-2 pmid: 19048258
10. Bakshi S, Sadhukhan A, Mishra S, Sahoo L. Improved Agrobacterium-mediated transformation of cowpea via sonication and vacuum infiltration. Plant Cell Rep. 2011;30(12):2281-2292. doi: 10.1007/s00299-0111133-8 pmid: 21853337

11. Chopra R, Aparna, Saini R. Use of sonication and vacuum infiltration for Agrobacterium - mediated transformation of an Indian lentil (Lens culinaris Medik.) cultivar. Sci Horticult. 2012; 143:127-134. doi: 10.1016/j.scienta.2012.06.019

12. Gasparis S, Bregier C, Orczyk W, Nadolska-Orczyk A. Agrobacterium-mediated transformation of oat (Avena sativa L.) cultivars via immature embryo and leaf explants. Plant Cell Rep. 2008;27(11):1721-1729. doi: 10.1007/s00299-008-0593-y pmid: 18690445

13. Murashige T, Skoog F. A Revised Medium for Rapid Growth and Bio Assays with Tobacco Tissue Cultures. Physiol Plant. 1962;15(3):473-497. doi: 10.1111/j.13 99-3054.1962.tb08052.x

14. Jefferson RA. Assaying chimeric genes in plants: The GUS gene fusion system. Plant Mol Biol Rep. 1987;5(4):387-405. doi: 10.1007/bf02667740

15. Saghai-Maroof MA, Soliman KM, Jorgensen RA, Allard RW. Ribosomal DNA spacer-length polymorphisms in barley: mendelian inheritance, chromosomal location, and population dynamics. Proc Natl Acad Sci U S A. 1984;81(24):8014-8018. doi: 10.1073/pnas.81.24.80 14 pmid: 6096873

16. Sapre S, Kumar S, Tiwari S. Transient GUS expression and efficient transformation in tobacco (Nicotiana tabacum). JNKVV Res J. 2011;45(1):96-100.

17. Trick HN, Finer JJ. Sonication-assisted Agrobacteriummediated transformation of soybean [Glycine max (L.) Merrill] embryogenic suspension culture tissue. Plant Cell Rep. 1998;17(6-7):482-488. doi: 10.1007/s002990 050429 pmid: 30736623

18. Gaba V, Kathiravan K, Amutha S, Singer S, Xiaodi X, Ananthakrishnan G. The Uses Of Ultrasound In Plant Tissue Culture. In: Dutta Gupta S, Ybaraki Y, editors. Plant tissue culture engineering. Netherlands: Springer; 2006. p. 417-426.

19. Dong J, Kharb P, Teng W, Hall TC. Characterization of rice transformed via an Agrobacterium-mediated inflorescence approach. Mol Breed. 2001;7(3):187-194. doi: 10.1023/a:1011357709073

20. Amoah BK, Wu H, Sparks C, Jones HD. Factors influencing Agrobacterium-mediated transient expression of uidA in wheat inflorescence tissue. $J$ Exp Bot. 2001;52(358):1135-1142. doi: 10.1093/jexbot/5 2.358.1135 pmid: 11432931 